\title{
A GREEDY HEURISTIC FOR A THREE-LEVEL MULTI-PERIOD SINGLE-SOURCING PROBLEM
}

\author{
H. Edwin Romeijn, Dolores Romero Morales
}

\begin{tabular}{|l|l|}
\hline \multicolumn{2}{|l|}{ ERIM REPORT SERIES RESEARCH IN MANAGEMENT } \\
\hline ERIM Report Series reference number & ERS-2000-04-LIS \\
\hline Publication status / version & draft / version January 2000 \\
\hline Number of pages & 16 \\
\hline Email address first author & Romeijn@ise.ufl.edu \\
\hline URL location (electronic version) & http://www.eur.nl/WebDOC/doc/erim/erimrs20000331144019.pdf \\
\hline Address & Erasmus Research Institute of Management (ERIM) \\
& Rotterdam School of Management / Faculteit Bedrijfskunde \\
& Erasmus Universiteit Rotterdam \\
& PoBox 1738 \\
& 3000 DR Rotterdam, The Netherlands \\
& Phone: \# 31-(0) 10-408 1182 \\
& Fax: \# 31-(0) 10-408 9020 \\
& Email: info@erim.eur.nl \\
& Internet: $\quad$ www.erim.eur.nl \\
\hline
\end{tabular}

Bibliographic data and classifications of all the ERIM reports are also available on the ERIM website: www.erim.eur.nl 


\author{
REPORT SERIES \\ RESEARCH IN MANAGEMENT
}

\begin{tabular}{|c|c|c|}
\hline \multicolumn{3}{|c|}{ BIBLIOGRAPHIC DATA AND CLASSIFICATIONS } \\
\hline Abstract & \multicolumn{2}{|c|}{$\begin{array}{l}\text { In this paper we consider a model for integrating transportation and inventory decisions in a } \\
\text { three-level logistics network consisting of plants, warehouses, and retailers (or customers). Our } \\
\text { model includes production and throughput capacity constraints, and minimizes production, } \\
\text { holding, and transportation costs in a dynamic environment. We show that the problem can be } \\
\text { reformulated as a certain type of assignment problem with convex objective function. Based on } \\
\text { this observation, we propose a greedy heuristic for the problem, and illustrate its behaviour on a } \\
\text { class of randomly generated problem instances. These experiments suggest that the heuristic } \\
\text { may be asymptotically feasible and optimal with probability one in the number of customers. }\end{array}$} \\
\hline \multirow{3}{*}{$\begin{array}{l}\text { Library of Congress } \\
\text { Classification } \\
\text { (LCC) }\end{array}$} & 5001-6182 & Business \\
\hline & $5001-6182$ & Business Science \\
\hline & $5761-5780$ & Shipping of Merchandise; Delivery of Goods \\
\hline \multirow{3}{*}{$\begin{array}{l}\text { Journal of Economic } \\
\text { Literature } \\
\text { (JEL) }\end{array}$} & M & Business Administration and Business Economics \\
\hline & $\begin{array}{l}\text { M } 11 \\
\text { R } 4\end{array}$ & $\begin{array}{l}\text { Production Management } \\
\text { Transportation Systems }\end{array}$ \\
\hline & R 49 & Transportation systems: other \\
\hline \multirow{3}{*}{$\begin{array}{l}\text { European Business Schools } \\
\text { Library Group } \\
\text { (EBSLG) }\end{array}$} & $85 \mathrm{~A}$ & Business General \\
\hline & $\begin{array}{l}260 \mathrm{~K} \\
240 \mathrm{~B}\end{array}$ & $\begin{array}{l}\text { Logistics } \\
\text { Information Systems Management }\end{array}$ \\
\hline & $260 \mathrm{~K}$ & Logistics \\
\hline \multicolumn{3}{|c|}{ Gemeenschappelijke Onderwerpsontsluiting (GOO) } \\
\hline \multirow[t]{4}{*}{ Classification GOO } & 85.00 & Bedriffskunde, Organisatiekunde: algemeen \\
\hline & 85.34 & Logistiek management \\
\hline & 85.20 & Bestuurlijke informatie, informatieverzorging \\
\hline & 85.34 & Logistiek management \\
\hline \multirow[t]{3}{*}{ Keywords G0O } & \multicolumn{2}{|c|}{ Bedrifskunde / Bedriffseconomie } \\
\hline & \multicolumn{2}{|c|}{ Bedrijfsprocessen, logistiek, management informatiesystemen } \\
\hline & \multicolumn{2}{|c|}{ Logistiek, voorraadbeheer, stochastische methoden } \\
\hline Free keywords & \multicolumn{2}{|c|}{$\begin{array}{l}\text { Dynamic Models, Dynamic Demand Pattern, Transportation Costs, Inventory Costs, Heuristic } \\
\text { Solution Approaches }\end{array}$} \\
\hline Other information & & \\
\hline
\end{tabular}




\title{
A greedy heuristic for a three-level multi-period single-sourcing problem
}

\author{
H. Edwin Romeijn* Dolores Romero Morales ${ }^{\dagger}$ \\ January 27, 2000
}

\begin{abstract}
In this paper we consider a model for integrating transportation and inventory decisions in a three-level logistics network consisting of plants, warehouses, and retailers (or customers). Our model includes production and throughput capacity constraints, and minimizes production, holding, and transportation costs in a dynamic environment. We show that the problem can be reformulated as a certain type of assignment problem with convex objective function. Based on this observation, we propose a greedy heuristic for the problem, and illustrate its behaviour on a class of randomly generated problem instances. These experiments suggest that the heuristic may be asymptotically feasible and optimal with probability one in the number of customers.
\end{abstract}

\section{Introduction}

The tendency to move towards global supply chains, the shortening for the product life cycle, and fast technological changes force companies to consider redesigning their logistics networks. The majority of the quantitative models proposed in the literature for the tactical problem of evaluating (usually with respect to costs) the layout of a distribution network assume a static environment. Hence the adequacy of those models is limited to situations where, in particular, the demand pattern is stationary over time. In addition, inventory decisions cannot be supported using stationary models.

In this paper we will study a multi-period single-sourcing problem (MPSSP) that can be used for evaluating logistics network designs with respect to costs in a dynamic environment. The logistics network consists of a set of plants, a set of warehouses, and a set of customers. For a given planning horizon, we assume that each plant has known, finite, and possibly time-varying capacity. Similarly, we consider that each warehouse has known, finite, and possibly time-varying throughput capacity. We assume that each

*Department of Industrial and Systems Engineering, University of Florida, 303 Weil Hall, P.O. Box 116595, Gainesville, Florida 32611-6595; email: romeijn@ise.ufl.edu.

${ }^{\dagger}$ Rotterdam School of Management, Erasmus University Rotterdam, P.O. Box 1738, 3000 DR Rotterdam, The Netherlands; e-mail: D.Romero@fbk.eur.nl. 
warehouse has essentially unlimited physical capacity. Moreover, the customers' demand patterns for a single product are assumed known. Finally, each customer needs to be delivered by (i.e., assigned to) a unique warehouse in each period. The decisions that need to be made are (i) production sites and quantities, (ii) assignment of customers to facilities, and (iii) location and size of inventories.

Since this problem is $\mathcal{N} \mathcal{P}$-Complete (see Martello and Toth [12] and Romero Morales, Van Nunen and Romeijn [17]), it is unlikely that efficient methods exist that can solve large problem instances to optimality. Therefore, it is appropriate to study heuristic approaches to this problem. We will show that our problem can be formulated as a certain type of assignment problem with convex objective function. This structure motivates the use of the class of greedy heuristics proposed by Martello and Toth [11] for the Generalized Assignment Problem (GAP), together with the family of pseudo-cost functions proposed by Romeijn and Romero Morales [14, 15, 16] for the GAP and two-level multi-period single-sourcing problems. Based on the structure of the LP-relaxation of our problem, we propose a suitable parameter choice, thereby identifying a particular greedy heuristic for the problem. We will provide numerical results on the performance of this heuristic, and conjecture that this member yields a heuristic that is asymptotically feasible and optimal in a probabilistic sense.

As mentioned above, related literature focuses mainly on static models. Examples are Geoffrion and Graves [9], Benders et al. [2], and Fleischmann [7]. Duran [6] studies a dynamic model for the planning of production, bottling, and distribution of beer, but focuses on the production process. Klose [10] analyzes the one-product version of the model proposed by Geoffrion and Graves [9]. Chan, Muriel and Simchi-Levi [3] study a dynamic, but uncapacitated, distribution problem in an operational setting. Arntzen et al. [1] present a multi-echelon multi-period model with no single-sourcing constraints on the assignment variables which was used in the reorganization of Digital Equipment Corporation.

The remainder of the paper is organized as follows. In Section 2 we will formulate the multi-period single-sourcing problem as a mixed-integer linear programming problem, and derive some properties of its LP-relaxation. In Section 3 we show the relationship with the GAP through a reformulation of the problem as a certain assignment problem with convex objective function. In Section 4 we will discuss a class of greedy heuristics for the problem, and select a suitable member of that class for which numerical experiments will be presented. The paper ends in Section 5 with some concluding remarks.

\section{The multi-period single-sourcing problem}

\subsection{A mixed-integer formulation}

Let $n$ denote the number of customers, $m$ the number of warehouses, $q$ the number of plants, and $T$ the number of time periods. The demand of customer $j$ in period $t$ is denoted by $d_{j t}$, while the production capacity at plant $l$ in period $t$ is equal to $b_{l t}$, and the maximal throughput capacity at warehouse $i$ in period $t$ is equal to $r_{i t}$. The production, handling and transportation costs per unit produced at plant $l$ and transported to warehouse $i$ in period $t$ are $c_{l i t}$. The costs of delivering the demand of customer $j$ from warehouse $i$ in 
period $t$ (i.e., the costs of assigning customer $j$ to warehouse $i$ in period $t$ ) are $a_{i j t}$. Each customer needs to be assigned to a single warehouse in any given period, which implies that the transportation costs can be an arbitrary (nonnegative) function of demand and distance. The inventory holding costs per unit at warehouse $i$ in period $t$ are $h_{i t}$. (Note that all parameters are required to be nonnegative.)

The multi-period single-sourcing problem (MPSSP) can now be formulated as follows:

$$
\operatorname{minimize} \sum_{t=1}^{T} \sum_{l=1}^{q} \sum_{i=1}^{m} c_{l i t} y_{l i t}+\sum_{t=1}^{T} \sum_{i=1}^{m} \sum_{j=1}^{n} a_{i j t} x_{i j t}+\sum_{t=1}^{T} \sum_{i=1}^{m} h_{i t} I_{i t}
$$

subject to

$$
\begin{array}{rlrl}
\sum_{j=1}^{n} d_{j t} x_{i j t}+I_{i t} & =\sum_{l=1}^{q} y_{l i t}+I_{i, t-1} \\
& i=1, \ldots, m ; t=1, \ldots, T \\
\sum_{i=1}^{m} y_{l i t} \leq b_{l t} & & l=1, \ldots, q ; t=1, \ldots, T \\
\sum_{j=1}^{n} d_{j t} x_{i j t} & \leq r_{i t} & & i=1, \ldots, m ; t=1, \ldots, T \\
I_{i 0} & =0 & & i=1, \ldots, m \\
\sum_{i=1}^{m} x_{i j t} & =1 & & j=1, \ldots, n ; t=1, \ldots, T \\
x_{i j t} & \in\{0,1\} & & i=1, \ldots, m ; j=1, \ldots, n ; t=1, \ldots, T \\
y_{l i t} & \geq 0 & l & =1, \ldots, q ; i=1, \ldots, m ; t=1, \ldots, T \\
I_{i t} & \geq 0 & i & =1, \ldots, m ; t=1, \ldots, T
\end{array}
$$

where $y_{l i t}$ is the amount produced at plant $l$ and delivered to warehouse $i$ in period $t$, $x_{i j t}$ is 1 if customer $j$ is assigned to warehouse $i$ in period $t$ and 0 otherwise, and $I_{i t}$ denotes the inventory level at warehouse $i$ at the end of period $t$. Constraints (1) impose the balance between the inflow, the storage and the outflow at warehouse $i$ in period $t$. The maximal production capacity at plant $l$ in period $t$ is restricted by (2) and the maximal throughput capacity at warehouse $i$ in period $t$ by constraint (3). Without loss of generality, we impose in (4) that the inventory level at the beginning of the planning horizon is equal to zero. Constraints (5) and (6) ensure that each customer is delivered by exactly one warehouse in each period.

This model extends the classical Single-Sourcing Problem (SSP) (see De Maio and Roveda [5]) in two directions. Firstly, the static character of the SSP prohibits the possibility of explicitly including decisions related to inventory management in the model. Secondly, the SSP assumes a layout of the distribution network where the production quantities are not included, or at least are not relevant (for instance when there is a one-to-one correspondence between warehouses and plants).

In the following section we will derive some properties of the LP-relaxation of the MPSSP. 


\subsection{Properties of the LP-relaxation of the MPSSP}

The LP-relaxation of $(\mathrm{P})$ can be formulated as follows:

$$
\operatorname{minimize} \sum_{t=1}^{T} \sum_{l=1}^{q} \sum_{i=1}^{m} c_{l i t} y_{l i t}+\sum_{t=1}^{T} \sum_{i=1}^{m} \sum_{j=1}^{n} a_{i j t} x_{i j t}+\sum_{t=1}^{T} \sum_{i=1}^{m} h_{i t} I_{i t}
$$

subject to

$$
\begin{array}{rlrl}
\sum_{j=1}^{n} d_{j t} x_{i j t}+I_{i t} & =\sum_{l=1}^{q} y_{l i t}+I_{i, t-1} \\
& i=1, \ldots, m ; t=1, \ldots, T \\
\sum_{i=1}^{m} y_{l i t} & \leq b_{l t} & l & \\
\sum_{j=1}^{n} d_{j t} x_{i j t} & \leq r_{i t} & & i=1, \ldots, q ; t=1, \ldots, T \\
I_{i 0} & =0 & & i=1, \ldots, m \\
\sum_{i=1}^{m} x_{i j t} & =1 & & j=1, \ldots, n ; t=1, \ldots, T \\
x_{i j t} & \geq 0 & & i=1, \ldots, m ; j=1, \ldots, n ; t=1, \ldots, T \\
y_{l i t} & \geq 0 & l & =1, \ldots, q ; i=1, \ldots, m ; t=1, \ldots, T \\
I_{i t} & \geq 0 & i & =1, \ldots, m ; t=1, \ldots, T .
\end{array}
$$

The following lemma derives a bound on the number of split assignments in the optimal solution for (LP). Let $B$ be the set of (customer-period)-pairs such that $(j, t) \in B$ means that customer $j$ is split in period $t$ (i.e., customer $j$ is assigned to more than one warehouse in period $t$, each satisfying part of its demand).

Lemma 2.1 The optimal solution for (LP) satisfies:

$$
|B| \leq 2 m T+q T \text {. }
$$

Proof: Rewrite the problem (LP) with equality constraints by introducing slack variables in the production capacity constraints (7) and in the throughput capacities constraints (8). We then obtain a problem with $m T+q T+m T+n T=2 m T+q T+n T$ equality constraints. Now consider the optimal solution to (LP). The number of variables having a nonzero value in this solution is no larger than the number of equality constraints in the reformulated problem. Since there is at least one nonzero assignment variable corresponding to each assignment constraint, and exactly one nonzero assignment variable corresponding to each assignment that is feasible with respect to the integrality constraints of $(\mathrm{P})$, there can be no more than $2 m T+q T$ assignments that are split. 
After eliminating the variables $I_{i 0}$ using equations (9), the dual of (LP) can be formulated as

$$
\operatorname{maximize} \sum_{t=1}^{T} \sum_{j=1}^{n} v_{j t}-\sum_{t=1}^{T} \sum_{l=1}^{q} b_{l t} \omega_{l t}-\sum_{t=1}^{T} \sum_{i=1}^{m} r_{i t} \nu_{i t}
$$

subject to

$$
\begin{array}{rlrl}
v_{j t} & \leq a_{i j t}+\lambda_{i t} d_{j t}+\nu_{i t} d_{j t} & & i=1, \ldots, m ; j=1, \ldots, n ; t=1, \ldots, T \\
\lambda_{i t} \leq \omega_{l t}+c_{l i t} & & i=1, \ldots, m ; l=1, \ldots, q ; t=1, \ldots, T \\
-\lambda_{i t}+\lambda_{i, t+1} \leq h_{i t} & & i=1, \ldots, m ; t=1, \ldots, T-1 \\
\lambda_{i t} & \text { free } & i & =1, \ldots, m ; t=1, \ldots, T \\
\omega_{l t} & \geq 0 & l & =1, \ldots, q ; t=1, \ldots, T \\
\nu_{i t} & \geq 0 & & i=1, \ldots, m ; t=1, \ldots, T \\
v_{j t} & \text { free } & & j=1, \ldots, n ; t=1, \ldots, T .
\end{array}
$$

The following proposition suggests a way to use the dual optimal solution to distinguish split assignments from non-split ones.

Proposition 2.2 Suppose that $(L P)$ is feasible and non-degenerate. Let $\left(x^{*}, y^{*}, I^{*}\right)$ be a basic optimal solution for $(L P)$ and let $\left(\lambda^{*}, \omega^{*}, \nu^{*}, v^{*}\right)$ be the corresponding optimal solution for (D). Then,

1. For each $(j, t) \notin B, x_{i j t}^{*}=1$ if and only if

$$
a_{i j t}+\lambda_{i t}^{*} d_{j t}+\nu_{i t}^{*} d_{j t}=\min _{k=1, \ldots, m}\left(a_{k j t}+\lambda_{k t}^{*} d_{j t}+\nu_{k t}^{*} d_{j t}\right)
$$

and

$$
a_{i j t}+\lambda_{i t}^{*} d_{j t}+\nu_{i t}^{*} d_{j t}<\min _{k=1, \ldots, m ; k \neq i}\left(a_{k j t}+\lambda_{k t}^{*} d_{j t}+\nu_{k t}^{*} d_{j t}\right) .
$$

2. For each $(j, t) \in B$, there exists an index $i$ such that

$$
a_{i j t}+\lambda_{i t}^{*} d_{j t}+\nu_{i t}^{*} d_{j t}=\min _{k=1, \ldots, m ; k \neq i}\left(a_{k j t}+\lambda_{k t}^{*} d_{j t}+\nu_{k t}^{*} d_{j t}\right) .
$$

Proof: See the Appendix.

\section{A convex assignment formulation for the MPSSP}

The MPSSP has been formulated as a mixed integer linear programming problem in the assignment, production and inventory variables. The throughput constraints (3) together with constraints (5) and (6) suggest a relationship between the MPSSP and the GAP. In fact, we will prove in this section that $(\mathrm{P})$ can be reformulated as a convex assignment problem in the variables $x$. The feasible region of this reformulation is formed by the Cartesian product of the feasible regions of $T$ SSPs, which are linked by the objective function, which is the sum of a linear and a convex function in $x$. 
The following lemma will be used in the proof of Proposition 3.2. The lemma derives a necessary and sufficient condition for the feasibility of a dynamic extension of the standard transportation problem. As in the standard transportation problem, the problem is to satisfy the demand at a set of demand points from a set of supply points. However, the extension lies in the dynamic nature of the problem, and the fact that early deliveries are allowed.

Hereafter, let $\mathbb{R}_{+}$denote the set of nonnegative numbers, i.e., $\mathbb{R}_{+}=[0,+\infty)$.

Lemma 3.1 The condition

$$
\sum_{\tau=1}^{t} \sum_{i=1}^{m} \delta_{i \tau} \leq \sum_{\tau=1}^{t} \sum_{l=1}^{q} \gamma_{l \tau} \quad \text { for each } t=1, \ldots, T
$$

is necessary and sufficient for the existence of a vector $y \in \mathbb{R}_{+}^{q m T}$ such that

$$
\sum_{i=1}^{m} y_{l i t} \leq \gamma_{l t} \quad l=1, \ldots, q ; t=1, \ldots, T
$$

and

$$
\sum_{\tau=1}^{t} \sum_{l=1}^{q} y_{l i \tau} \geq \sum_{\tau=1}^{t} \delta_{i \tau} \quad i=1, \ldots, m ; t=1, \ldots, T .
$$

Proof: It can easily be seen that condition (10) is necessary to ensure the existence of a nonnegative vector $y \in \mathbb{R}_{+}^{q m T}$ satisfying (11) and (12). We will show, by induction on $t$, that this condition is also sufficient.

For $t=1$, the inequalities in conditions (11) and (12) together with the nonnegativity assumption on $y$ define the feasible region of a standard transportation problem. Moreover, the inequality in condition (10) for $t=1$ says that the aggregate demand cannot exceed the aggregate capacity, which clearly is a sufficient condition for feasibility of the standard transportation problem.

Now, we will assume that if the inequality conditions in (10) hold for $t=1, \ldots, t^{\prime}$, then there exists a nonnegative vector $y \in \mathbb{R}_{+}^{q m t^{\prime}}$ so that the inequalities in conditions (11) and (12) are satisfied for $t=1, \ldots, t^{\prime}$. We will show that the same result holds for horizon $t^{\prime}+1$.

We will distinguish two cases, depending on the difference between the aggregate demand in period $t^{\prime}+1$ and the aggregate capacity in the same period. First consider the case where the aggregate demand is no more than the aggregate capacity, i.e.,

$$
\sum_{i=1}^{m} \delta_{i, t^{\prime}+1} \leq \sum_{l=1}^{q} \gamma_{l, t^{\prime}+1}
$$

Then there exists a vector $z \in \mathbb{R}_{+}^{q m}$ such that

$$
\sum_{i=1}^{m} z_{l i, t^{\prime}+1} \leq \gamma_{l, t^{\prime}+1} \quad l=1, \ldots, q
$$

and 


$$
\sum_{l=1}^{q} z_{l i, t^{\prime}+1} \geq \delta_{i, t^{\prime}+1} \quad i=1, \ldots, m .
$$

Moreover, by the induction hypothesis there exists a vector $y \in \mathbb{R}_{+}^{q m t^{\prime}}$ such that

$$
\sum_{i=1}^{m} y_{l i t} \leq \gamma_{l t} \quad l=1, \ldots, q ; t=1, \ldots, t^{\prime}
$$

and

$$
\sum_{\tau=1}^{t} \sum_{l=1}^{q} y_{l i \tau} \geq \sum_{\tau=1}^{t} \delta_{i \tau} \quad i=1, \ldots, m ; t=1, \ldots, t^{\prime} .
$$

It is easy to see that $(y, z)$ a nonnegative vector satisfying the inequalities in conditions (11) and (12) for $t=1, \ldots, t^{\prime}+1$.

Next, we will consider the case where

$$
\sum_{i=1}^{m} \delta_{i, t^{\prime}+1}>\sum_{l=1}^{q} \gamma_{l, t^{\prime}+1}
$$

It suffices to show that the excess demand in period $t^{\prime}+1$, i.e.,

$$
\sum_{i=1}^{m} \delta_{i, t^{\prime}+1}-\sum_{l=1}^{q} \gamma_{l, t^{\prime}+1}
$$

can be supplied in previous periods. This is easy to see since

$$
\sum_{\tau=1}^{t} \sum_{i=1}^{m} \delta_{i \tau} \leq \sum_{\tau=1}^{t} \sum_{l=1}^{q} \gamma_{l \tau} \quad t=1, \ldots, t^{\prime}-1
$$

and

$$
\sum_{\tau=1}^{t^{\prime}} \sum_{i=1}^{m} \delta_{i \tau}+\sum_{i=1}^{m} \delta_{i, t^{\prime}+1}-\sum_{l=1}^{q} \gamma_{l, t^{\prime}+1} \leq \sum_{\tau=1}^{t^{\prime}} \sum_{l=1}^{q} \gamma_{l \tau}
$$

Proposition 3.2 Problem $(P)$ can be reformulated as:

$$
\text { minimize } \sum_{t=1}^{T} \sum_{i=1}^{m} \sum_{j=1}^{n} a_{i j t} x_{i j t}+H(x)
$$

subject to

$$
\begin{array}{rlrl}
\sum_{j=1}^{n} d_{j t} x_{i j t} \leq r_{i t} & i=1, \ldots, m ; t=1, \ldots, T \\
\sum_{i=1}^{m} x_{i j t}=1 & j=1, \ldots, n ; t=1, \ldots, T \\
x_{i j t} & \in\{0,1\} & i=1, \ldots, m ; j=1, \ldots, n ; t=1, \ldots, T
\end{array}
$$


where $H(x)$ is the convex function given by the optimal value of the following linear problem:

$$
\text { minimize } \sum_{t=1}^{T} \sum_{l=1}^{q} \sum_{i=1}^{m} c_{l i t} y_{l i t}+\sum_{t=1}^{T} \sum_{i=1}^{m} h_{i t} I_{i t}
$$

subject to

$$
\begin{array}{rlrl}
I_{i, t-1}-I_{i t}+\sum_{l=1}^{q} y_{l i t} & =\sum_{j=1}^{n} d_{j t} x_{i j t} & & i=1, \ldots, m ; t=1, \ldots, T \\
\sum_{i=1}^{m} y_{l i t} & \leq b_{l t} & & l=1, \ldots, q ; t=1, \ldots, T \\
I_{i 0} & =0 & & i=1, \ldots, m \\
y_{l i t} & \geq 0 & l & l=1, \ldots, q ; i=1, \ldots, m ; t=1, \ldots, T \\
I_{i t} & \geq 0 & & i=1, \ldots, m ; t=1, \ldots, T .
\end{array}
$$

Proof: The result follows by a decomposition argument. Let $\mathcal{F}$ be the feasible region of (P). We then have that

$$
\begin{aligned}
\min _{(x, y, I) \in \mathcal{F}}\left(\sum_{t=1}^{T} \sum_{l=1}^{q} \sum_{i=1}^{m} c_{l i t} y_{l i t}+\sum_{t=1}^{T} \sum_{i=1}^{m} \sum_{j=1}^{n} a_{i j t} x_{i j t}+\sum_{t=1}^{T} \sum_{i=1}^{m} h_{i t} I_{i t}\right)= \\
=\min _{x: \exists\left(y^{\prime}, I^{\prime}\right)\left(x, y^{\prime}, I^{\prime}\right) \in \mathcal{F}}\left(\sum_{t=1}^{T} \sum_{i=1}^{m} \sum_{j=1}^{n} a_{i j t} x_{i j t}+\right. \\
\left.\min _{(y, I):(x, y, I) \in \mathcal{F}}\left(\sum_{t=1}^{T} \sum_{l=1}^{q} \sum_{i=1}^{m} c_{l i t} y_{l i t}+\sum_{t=1}^{T} \sum_{i=1}^{m} h_{i t} I_{i t}\right)\right) \\
=\min _{x: \exists\left(y^{\prime}, I^{\prime}\right)\left(x, y^{\prime}, I^{\prime}\right) \in \mathcal{F}}\left(\sum_{t=1}^{T} \sum_{i=1}^{m} \sum_{j=1}^{n} a_{i j t} x_{i j t}+H(x)\right) .
\end{aligned}
$$

Observe that

$$
\sum_{\tau=1}^{t} \sum_{j=1}^{n} d_{j \tau} \leq \sum_{\tau=1}^{t} \sum_{l=1}^{q} b_{l \tau} \quad \text { for each } t=1, \ldots, T
$$

is a necessary condition for feasibility for both $\left(\mathrm{P}^{\prime}\right)$ and the decomposed problem. Thus, hereafter we will assume that condition (15) holds. It remains to be shown that

$$
\mathcal{F}^{\prime} \equiv\left\{x \in \mathbb{R}^{m n T}: \exists(y, I) \in \mathbb{R}^{q m T} \times \mathbb{R}^{m T} \text { such that }(x, y, I) \in \mathcal{F}\right\}
$$

is the feasible region of $\left(\mathrm{P}^{\prime}\right)$.

It is obvious that $\mathcal{F}^{\prime}$ is contained in the feasible region of $\left(\mathrm{P}^{\prime}\right)$. Now let $x$ be a feasible vector for $\left(\mathrm{P}^{\prime}\right)$. Lemma 3.1 and condition (15) imply that there exists a vector $y \in \mathbb{R}_{+}^{q m T}$ such that

$$
\sum_{i=1}^{m} y_{l i t} \leq b_{l t} \quad l=1, \ldots, q ; t=1, \ldots, T
$$

and 


$$
\sum_{\tau=1}^{t} \sum_{l=1}^{q} y_{l i \tau} \geq \sum_{\tau=1}^{t} \sum_{j=1}^{n} d_{j \tau} x_{i j \tau} \quad i=1, \ldots, m ; t=1, \ldots, T .
$$

Now define $I_{i t}$, for $i=1, \ldots, m$ and $t=1, \ldots, T$, as

$$
I_{i t}=\sum_{\tau=1}^{t} \sum_{l=1}^{q} y_{l i \tau}-\sum_{\tau=1}^{t} \sum_{j=1}^{n} d_{j \tau} x_{i j \tau}
$$

It is easy to see that $I_{i t}$ is nonnegative and $(x, y, I) \in \mathcal{F}$, and thus $x \in \mathcal{F}^{\prime}$.

Using strong duality for linear programming, it is straightforward to show that the function $H$ is convex.

This result shows that, for each assignment solution to $\left(\mathrm{P}^{\prime}\right)$, corresponding optimal values for the production and inventory variables exist. A similar result was derived by Freling et al. [8], for the case where there exists a one to one correspondence between warehouses and plants. In this case, the objective function of the assignment problem is separable in the index $i$. The separability of the objective function allows the reformulation of the problem as a set partitioning problem, which can be used to construct a Branch and Price algorithm for this class of problems.

\section{Solving the MPSSP}

\subsection{A greedy heuristic for the MPSSP}

In the previous section we have shown that the MPSSP can be formulated as a collection of $T$ SSP's that are joined through a convex objective function. Since the Single Sourcing Problem is a special case of the GAP, we propose to use a greedy heuristic similar to the one proposed by Martello and Toth [11] for the GAP, using a pseudo-cost function from the family introduced by Romeijn and Romero Morales [14].

The idea of the heuristic is that each possible assignment of a (customer,period)-pair $(j, t)$ to a warehouse $i$ is evaluated by a pseudo-cost function $f(i, j, t)$. For each assignment to be made, the difference between the two smallest values of $f(i, j, t)$ (called the desirability of making the cheapest assignment with respect to the pseudo-cost) is computed, and assignments are made in decreasing order of this difference. Along the way, the remaining capacities of the warehouses, and consequently the values of the desirabilities, are updated to ensure feasibility. Note, from formulation $\left(\mathrm{P}^{\prime}\right)$ of the MPSSP, that only the throughput capacities play a role with respect to feasibility.

Romeijn and Romero Morales [14] propose to use the following family of pseudo-cost functions:

$$
f(i, j, t)=a_{i j t}+\alpha_{i t} d_{j t}
$$

where $\alpha \in \mathbb{R}_{+}^{m T}$. We may observe that this pseudo-cost function combines costs $\left(a_{i j t}\right)$ with demands $\left(d_{j t}\right)$ (i.e., the use of the scarce throughput capacity at the warehouses). The result of Proposition 2.2, where the split and nonsplit assignments in the LP-relaxation of 
(P) are characterized, suggests using the following member of the family of pseudo-cost functions:

$$
f(i, j, t)=a_{i j t}+\left(\lambda_{i t}^{*}+\nu_{i t}^{*}\right) d_{j t}
$$

where $\lambda^{*} \in \mathbb{R}_{+}^{m T}$ is the vector of optimal dual multipliers of the flow conservation constraints in (LP), and similarly $\nu^{*} \in \mathbb{R}_{+}^{m T}$ is the vector of optimal dual multipliers of the throughput constraints (where the corresponding constraints are reformulated as $\geq$ constraints, so that the dual multipliers are nonnegative). A probabilistic analysis of such heuristics on similar problems leads us to conjecture that this choice will yield a heuristic that is asymptotically optimal in a probabilistic sense (as $n$ goes to $\infty$ ).

\subsection{Some numerical results}

In this section we will illustrate the behaviour of the greedy heuristic as described in the previous section on a set of randomly generated test problems. For each problem instance, we generate a set of $n$ customers, a set of $m$ warehouses, and a set of $q$ plants uniformly in the square $[0,10]^{2}$. For customer $j(j=1, \ldots, n)$, we generate a random demand $D_{j t}$ in period $t(t=1, \ldots, T)$ from the uniform distribution on $\left[5 \sigma_{t}, 25 \sigma_{t}\right]$, where the vector $\sigma$ contains seasonal factors, which we have chosen to be $\sigma=\left(\frac{1}{2}, \frac{3}{4}, 1,1, \frac{3}{4}, \frac{1}{2}\right)^{\top}$. The production costs are assumed to be equal to the distance, i.e., $c_{l i t}=\operatorname{dist}_{l i}$, where dist $_{l i}$ denotes the Euclidean distance between plant $l$ and warehouse $i$. The assignment costs are assumed to be proportional to demand and distance, i.e., $a_{i j t}=d_{j t} \cdot$ dist $_{i j}$, where dist $_{i j}$ denotes the Euclidean distance between warehouse $i$ and customer $j$. Finally, we generate inventory holding costs $H_{i t}$ uniformly from [10,30].

We have chosen the capacities equal to $b_{l t}=\frac{1}{q} \cdot \beta \cdot n$ and $r_{i t}=\frac{1}{m} \cdot \rho \cdot n$, where

$$
\begin{aligned}
& \beta=\delta \cdot 15 \cdot \max _{t=1, \ldots, T}\left(\frac{1}{t} \sum_{\tau=1}^{t} \sigma_{\tau}\right) \\
& \rho=\delta \cdot 15 \cdot \max _{t=1, \ldots, T} \sigma_{t} .
\end{aligned}
$$

The results of Lemma 3.1 and Romeijn and Piersma [13] show that the instances generated by this probabilistic model are asymptotically feasible with probability one (as $n$ goes to $\infty$ ) if $\delta>1$, and infeasible with probability one (again as $n$ goes to $\infty$ ) if $\delta<1$. To account for the asymptotic nature of this feasibility guarantee, we have set $\delta=1.1$ to obtain feasible instances for finite $n$.

We have fixed the number of plants at $q=3$, the number of warehouses at $m=5$, and the number of periods at $T=6$. We let the number of customers vary from $n=50$ till $n=500$ in increments of 50 customers. For each class of instances and each size of the problem we have generated 50 instances. All the runs were performed on a PC with a $350 \mathrm{MHz}$ Pentium II processor and $128 \mathrm{MB}$ RAM. All LP-relaxations were solved using CPLEX 6.5 [4].

Table 1 illustrates the behaviour of the greedy heuristic (using the pseudo-cost function mentioned in Section 4.1). Clearly, $n$ denotes the number of customers. The table shows the number of instances for which the LP-relaxation was feasible, as well as the number of instances for which the heuristic found a feasible solution. In addition, the time needed 
to solve the LP-relaxation, as well as the total time needed to find the heuristic solution (i.e., including the time needed to solve the LP-relaxation) is shown. Finally, an upper bound on the average error of the heuristic solution is shown, as measured by the relative deviation of the heuristic solution value from the optimal LP-value. This average was calculated only using the instances where the heuristic found a feasible solution.

\begin{tabular}{|r|cc|ccc|}
\hline & \multicolumn{2}{|c|}{ LP } & \multicolumn{3}{c|}{ heuristic } \\
\hline$n$ & \# feasible & time (sec.) & \# feasible & time (sec.) & error (\%) \\
\hline \hline 50 & 42 & 0.17 & 35 & 0.21 & 1.26 \\
100 & 48 & 0.42 & 47 & 0.46 & 0.59 \\
150 & 50 & 0.68 & 50 & 0.74 & 0.42 \\
200 & 50 & 1.20 & 50 & 1.26 & 0.28 \\
250 & 50 & 1.80 & 50 & 1.87 & 0.24 \\
300 & 50 & 2.34 & 50 & 2.42 & 0.28 \\
350 & 50 & 2.90 & 50 & 2.98 & 0.16 \\
400 & 50 & 3.37 & 50 & 3.46 & 0.17 \\
450 & 50 & 3.91 & 50 & 4.00 & 0.12 \\
500 & 50 & 4.83 & 50 & 4.93 & 0.12 \\
\hline
\end{tabular}

Table 1: Greedy heuristic

Although we cannot guarantee that the heuristic will always find a feasible solution (recall that even to determine whether a particular instance of the MPSSP is feasible is an $\mathcal{N} \mathcal{P}$-complete problem), a feasible solution was always found for instances with at least 150 customers. Note that feasibility of the LP-relaxation does not imply feasibility of the MPSSP, so that the inability of the heuristic to find a feasible solution could be caused by infeasibility of the instance, even when the LP-relaxation is feasible.

Except for the smallest class of instances, the average error was always well below $1 \%$. Moreover, the fact that the average error decreases as the number of customers increases supports our conjecture that the heuristic is asymptotically optimal.

In addition to using the heuristic, we have also used the MIP solver of CPLEX to try to solve the problems to optimality for the two smallest problem sizes. The procedure was cut after 30 minutes, which happened for 12 problem instances with 50 customers, and for 29 instances with 100 customers. For the instances with 50 customers, the average time spent by CPLEX was 525 seconds (and 122 seconds when we disregard the most difficult instances where the optimal solution was not found within 30 minutes). For the instances with 100 customers, these numbers were 1200 and 362, respectively. Comparing these to the times spent by the heuristic, we conclude that the heuristic is a very effective way of finding a high quality solution with little effort.

\section{Conclusions}

In this paper we have analyzed a model for evaluating the design of a logistics network in a dynamic environment. The network consists of plants, warehouses and customers. 
The model deals with production and throughput constraints, as well as standard singlesourcing constraints. Based on a reformulation of the problem as a convex assignment problem, we have proposed a greedy heuristic. The numerical illustrations indicate that the heuristic may be asymptotically feasible and optimal.

\section{References}

[1] B.C. Arntzen, G.G. Brown, T.P. Harrison, and L.L. Trafton. Global supply chain management at Digital Equipment Corporation. Interfaces, 25(1):69-93, 1995.

[2] J.F. Benders, J.A. Keulemans, J.A.E.E. van Nunen, and G. Stolk. A decision support program for planning locations and allocations with the aid of linear programming. In C.B. Tilanus, O.B. de Gaus, and J.K. Lenstra, editors, Quantitative Methods in Management: cases studies of failures and successes, chapter 4, pages 29-34. John Wiley \& Sons, Chichester, 1986.

[3] L.M.A. Chan, A. Muriel, and D. Simchi-Levi. Supply-Chain Management: Integrating inventory and transportation. Research Report, Department of Industrial Engeneering and Management Sciences, Northwestern University, Evanston, Illinois, 1998.

[4] CPLEX Reference Manual. ILOG CPLEX 6.5. ILOG, Inc., Incline Village, Nevada, 1999.

[5] A. De Maio and C. Roveda. An all zero-one algorithm for a certain class of transportation problems. Operations Research, 19(6):1406-1418, 1971.

[6] F. Duran. A large mixed integer production and distribution program. European Journal of Operational Research, 28:207-217, 1987.

[7] B. Fleischmann. Designing distribution systems with transport economies of scale. European Journal of Operational Research, 70:31-42, 1993.

[8] R. Freling, H.E. Romeijn, D. Romero Morales, and A.P.M. Wagelmans. A branch and price algorithm for the multi-period single-sourcing problem. ERASM Management Report Series no. 49-1999, Rotterdam School of Management, Erasmus University Rotterdam, 1999.

[9] A. Geoffrion and G.W. Graves. Multicommodity distribution system design by Benders decomposition. Management Science, 20(5):822-844, 1974.

[10] A. Klose. An LP-based heuristic for two-stage capacitated facility location problems. Journal of the Operational Research Society, 50:157-166, 1999.

[11] S. Martello and P. Toth. An algorithm for the generalized assignment problem. In J.P. Brans, editor, Operational Research '81, pages 589-603. IFORS, North-Holland, Amsterdam, 1981. 
[12] S. Martello and P. Toth. Knapsack problems, algorithms and computer implementations. John Wiley \& Sons, New York, 1990.

[13] H.E. Romeijn and N. Piersma. A probabilistic feasibility and value analysis of the generalized assignment problem. ERASM Management Report Series no. 293, Rotterdam School of Management, Erasmus University Rotterdam, 1996.

[14] H.E. Romeijn and D. Romero Morales. A class of greedy algorithms for the generalized assignment problem. ERASM Management Report Series no. 40(13), Rotterdam School of Management, Erasmus University Rotterdam, 1997. Forthcoming in Discrete Applied Mathematics.

[15] H.E. Romeijn and D. Romero Morales. Asymptotic analysis of a greedy heuristic for the multi-period single-sourcing problem: the acyclic case. Research Report 99-13, Department of Industrial and Systems Engineering, University of Florida, 1999.

[16] H.E. Romeijn and D. Romero Morales. An asymptotically optimal greedy heuristic for the multi-period single-sourcing problem: the cyclic case. ERASM Management Report Series no. 20-1999, Rotterdam School of Management, Erasmus University Rotterdam, 1999.

[17] D. Romero Morales, J.A.E.E. van Nunen, and H.E. Romeijn. Logistics network design evaluation in a dynamic environment. In M.G. Speranza and P. Stähly, editors, New trends in distribution logistics, Lecture notes in economics and mathematical systems 480, pages 113-135. Springer-Verlag, Berlin, 1999.

\section{Appendix}

Let $\left(x^{*}, y^{*}, I^{*}\right)$ be a basic optimal solution for (LP). In the following lemma, which will be used in the proof of Proposition 2.2, we derive a relationship between the number of split assignments, the number of fractional assignment variables, the number of (plant,warehouse,period)-triples having a positive flow, the number of (plant,period)-pairs where the plant is used to full capacity in that period, the number of (warehouse,period)pairs where the warehouse is used to full capacity in that period and the number of strictly positive inventory variables. Let $F$ be the set of fractional assignment variables, $Q$ the set of (plant,period)-pairs where the plant is used to full capacity in that period, $W$ the set of (warehouse,period)-pairs where the warehouse is used to full capacity in that period, $Y^{+}$the set of (plant,warehouse,period)-triples having a positive flow and $I^{+}$the set of strictly positive inventory variables, i.e.

$$
\begin{aligned}
F & =\left\{(i, j, t): 0<x_{i j t}^{*}<1\right\} \\
Q & =\left\{(l, t): \sum_{i=1}^{m} y_{l t}^{*}=b_{l t}\right\} \\
W & =\left\{(i, t): \sum_{j=1}^{n} d_{j t} x_{i j t}^{*}=r_{i t}\right\}
\end{aligned}
$$




$$
\begin{aligned}
Y^{+} & =\left\{(l, i, t): y_{\text {lit }}^{*}>0\right\} \\
I^{+} & =\left\{(i, t): I_{i t}^{*}>0\right\} .
\end{aligned}
$$

Lemma A.1 If (LP) is non-degenerate, then for a basic optimal solution of (LP) we have

$$
|F|+\left|Y^{+}\right|+\left|I^{+}\right|=m T+|Q|+|W|+|B| .
$$

Proof: Denote by $s_{l t}$ the slack variables corresponding to the production capacity constraints in (LP) and $S_{i t}$ the slack variables corresponding to the throughput capacity constraints. Thus, including these variables, (LP) can be reformulated as

$$
\operatorname{minimize} \sum_{t=1}^{T} \sum_{l=1}^{q} \sum_{i=1}^{m} c_{l i t} y_{l i t}+\sum_{t=1}^{T} \sum_{i=1}^{m} \sum_{j=1}^{n} a_{i j t} x_{i j t}+\sum_{t=1}^{T} \sum_{i=1}^{m} h_{i t} I_{i t}
$$

subject to

$$
\begin{array}{rlrl}
\sum_{j=1}^{n} d_{j t} x_{i j t}+I_{i t} & =\sum_{l=1}^{q} y_{l i t}+I_{i, t-1} \\
& & i=1, \ldots, m ; t=1, \ldots, T \\
\sum_{i=1}^{m} y_{l i t}+s_{l t} & =b_{l t} \quad & & l=1, \ldots, q ; t=1, \ldots, T \\
\sum_{j=1}^{n} d_{j t} x_{i j t}+S_{i t} & =r_{i t} & & i=1, \ldots, m ; t=1, \ldots, T \\
I_{i 0} & =0 & & i=1, \ldots, m \\
\sum_{i=1}^{m} x_{i j t} & =1 & & j=1, \ldots, n ; t=1, \ldots, T \\
x_{i j t} & \geq 0 & & i=1, \ldots, m ; j=1, \ldots, n ; t=1, \ldots, T \\
y_{l i t} & \geq 0 & & l=1, \ldots, q ; i=1, \ldots, m ; t=1, \ldots, T \\
I_{i t} & \geq 0 & & i=1, \ldots, m ; t=1, \ldots, T \\
s_{l t} & \geq 0 & & l=1, \ldots, q ; t=1, \ldots, T \\
S_{i t} & \geq 0 & & i=1, \ldots, m ; t=1, \ldots, T .
\end{array}
$$

Let $\left(x^{*}, y^{*}, I^{*}, s^{*}, S^{*}\right)$ be a basic optimal solution for (LP). Then, sets $Q$ and $W$, defined above, are equal to

$$
\begin{aligned}
Q & =\left\{(l, t): s_{l t}^{*}=0\right\} \\
W & =\left\{(i, t): S_{i t}^{*}=0\right\} .
\end{aligned}
$$

Under non-degeneracy, the number of nonzero variables at $\left(x^{*}, y^{*}, I^{*}, s^{*}, S^{*}\right)$ is equal to $2 m T+q T+n T$, the number of constraints in (LP). The number of nonzero assignment variables is equal to $(n T-|B|)+|F|$, where the first term corresponds to the variables 
$x_{i j t}^{*}=1$, the second one to the fractional assignment variables. With respect to the slack variables, we have $(q T-|Q|)+(m T-|W|)$ nonzero variables. By definition $\left|Y^{+}\right|$is the number of nonzero production variables. The same follows for $I^{+}$. Thus, by imposing that the number of nonzero variables at $\left(x^{*}, y^{*}, I^{*}, s^{*}, S^{*}\right)$ is equal to $2 m T+q T+n T$, we obtain

$$
2 m T+q T+n T=(n T-|B|)+|F|+(q T-|Q|)+(m T-|W|)+\left|Y^{+}\right| .
$$

The desired result now follows from the last equality.

Proposition 2.2 Suppose that (LP) is feasible and non-degenerate. Let $\left(x^{*}, y^{*}, I^{*}\right)$ be a basic optimal solution for $(L P)$ and let $\left(\lambda^{*}, \omega^{*}, \nu^{*}, v^{*}\right)$ be the corresponding optimal solution for (D). Then,

1. For each $(j, t) \notin B, x_{i j t}^{*}=1$ if and only if

$$
a_{i j t}+\lambda_{i t}^{*} d_{j t}+\nu_{i t}^{*} d_{j t}=\min _{k=1, \ldots, m}\left(a_{k j t}+\lambda_{k t}^{*} d_{j t}+\nu_{k t}^{*} d_{j t}\right)
$$

and

$$
a_{i j t}+\lambda_{i t}^{*} d_{j t}+\nu_{i t}^{*} d_{j t}<\min _{k=1, \ldots, m ; k \neq i}\left(a_{k j t}+\lambda_{k t}^{*} d_{j t}+\nu_{k t}^{*} d_{j t}\right) .
$$

2. For each $(j, t) \in B$, there exists an index $i$ such that

$$
a_{i j t}+\lambda_{i t}^{*} d_{j t}+\nu_{i t}^{*} d_{j t}=\min _{k=1, \ldots, m ; k \neq i}\left(a_{k j t}+\lambda_{k t}^{*} d_{j t}+\nu_{k t}^{*} d_{j t}\right) .
$$

Proof: Observe that

$$
\lambda_{i t}^{*}=\min _{l=1, \ldots, q}\left(c_{l i t}+\omega_{l t}\right) \geq 0 \quad \text { for } j=1, \ldots, n ; t=1, \ldots, T
$$

and by using the nonnegativity of vector $\lambda^{*}$ we have that

$$
v_{j t}^{*}=\min _{i=1, \ldots, m}\left(a_{i j t}+\lambda_{i t}^{*} d_{j t}+\nu_{i t}^{*} d_{j t}\right) \geq 0 \quad \text { for } j=1, \ldots, n ; t=1, \ldots, T .
$$

Thus, without loss of optimality, we can add to (D) the nonnegativity constraints on the vectors $\lambda$ and $v$. By adding slack variables $s_{i j t}, S_{l i t}$ and $U_{i t}$ to the constraints in (D), we can reformulate it as

$$
\operatorname{maximize} \sum_{t=1}^{T} \sum_{j=1}^{n} v_{j t}-\sum_{t=1}^{T} \sum_{l=1}^{q} b_{l t} \omega_{l t}-\sum_{t=1}^{T} \sum_{i=1}^{m} r_{i t} \nu_{i t}
$$

subject to

$$
\begin{array}{rlrl}
v_{j t}+s_{i j t} & =a_{i j t}+\lambda_{i t} d_{j t}+\nu_{i t} d_{j t} & & i=1, \ldots, m ; j=1, \ldots, n ; t=1, \ldots, T \\
\lambda_{i t}+S_{l i t} & =\omega_{l t}+c_{l i t} & i & =1, \ldots, m ; l=1, \ldots, q ; t=1, \ldots, T \\
-\lambda_{i t}+\lambda_{i, t+1}+U_{i t} & =h_{i t} & i & =1, \ldots, m ; t=1, \ldots, T-1 \\
\lambda_{i t} & \geq 0 & i & =1, \ldots, m ; t=1, \ldots, T
\end{array}
$$




$$
\begin{array}{r}
\omega_{l t} \geq 0 \\
\nu_{i t} \geq 0 \\
v_{j t} \geq 0 \\
s_{i j t} \geq 0 \\
S_{l i t} \geq 0 \\
U_{i t} \geq 0
\end{array}
$$

$$
\begin{aligned}
& l=1, \ldots, q ; t=1, \ldots, T \\
& i=1, \ldots, m ; t=1, \ldots, T \\
& j=1, \ldots, n ; t=1, \ldots, T \\
& i=1, \ldots, m ; j=1, \ldots, n ; t=1, \ldots, T \\
& l=1, \ldots, q ; i=1, \ldots, m ; t=1, \ldots, T \\
& i=1, \ldots, m ; t=1, \ldots, T-1 .
\end{aligned}
$$

Let $\left(\lambda^{*}, \omega^{*}, \nu^{*}, v^{*}, s^{*}, S^{*}, U^{*}\right)$ be the optimal solution for $\left(\mathrm{D}^{\prime}\right)$. For each $(j, t) \in B$, there exist at least two variables $x_{i j t}^{*}$ that are strictly positive. Hence, by the complementary slackness conditions, there exist at least two variables $s_{i j t}^{*}$ equal to zero. This proves Claim 2.

To prove Claim 1, it is enough to show that for each $(j, t) \notin B$ there exists exactly one variable $s_{i j t}^{*}=0$. By complementary slackness conditions we know that at least there exists one of these variables. We have to show the uniqueness, and we do it by counting the variables at level zero in the vector $\left(\lambda^{*}, \omega^{*}, \nu^{*}, v^{*}, s^{*}, S^{*}, U^{*}\right)$. There are at least $q T-|Q|$ variables $\omega_{l t}^{*}, m T-|W|$ variables $\nu_{i t}^{*},|F|$ variables $s_{i j t}^{*}$ corresponding to $(j, t) \in B, n T-|B|$ variables $s_{i j t}^{*}$ corresponding to $(j, t) \notin B,\left|Y^{+}\right|$variables $S_{l i t}^{*}$ equal to zero, and $\left|Y^{+}\right|$variables $U_{i t}^{*}$ equal to zero. In total, we have at least $q T-|Q|+m T-$ $|W|+|F|+n T-|B|+\left|Y^{+}\right|+\left|I^{+}\right|=q T+2 m T+n T$ zeroes in the optimal dual solution, where the last equality follows from Lemma A.1. So, these are exactly all the variables at level zero in vector $\left(\lambda^{*}, \omega^{*}, \nu^{*}, v^{*}, s^{*}, S^{*}, U^{*}\right)$. Then, for each $(j, t) \notin B$ there exists exactly one variable $s_{i j t}^{*}=0$, and Claim 1 follows. 


\section{ERASMUS RESEARCH INSTITUTE OF MANAGEMENT}

\section{REPORT SERIES RESEARCH IN MANAGEMENT}

Other Publications in the Report Series Research* in Management:

Impact of the Employee Communication and Perceived External Prestige on Organizational Identification, Ale Smidts, Cees B.M. van Riel \& Ad Th.H. Pruyn

ERS-2000-01-MKT

Critical Complexities, from marginal paradigms to learning networks,

Slawomir Magala

ERS-2000-02-ORG

Forecasting Market Shares from Models for Sales,

Dennis Fok \& Philip Hans Franses

ERS-2000-03-MKT

ERIM Research Programs:

LIS Business Processes, Logistics and Information Systems

ORG Managing Relationships for Performance

MKT Decision Making in Marketing Management

F\&A Financial Decision Making and Accounting

STR Strategic Renewal and the Dynamics of Firms, Networks and Industries 